\title{
A Method for Segmenting Bronchial Trees from 3D Chest X-ray CT Images
}

\author{
Takayuki Kitasaka ${ }^{1}$, Kensaku Mori ${ }^{1}$, Yasuhito Suenaga ${ }^{1}$, Jun-ichi Hasegawa ${ }^{2}$, \\ and Jun-ichiro Toriwaki ${ }^{2}$ \\ 1 Graduate School of Information Science, Nagoya University, \\ Furo-cho, Chikusa-ku, Nagoya, Aichi, 464-8603, Japan \\ \{kitasaka, mori, suenaga\}@is.nagoya-u.ac.jp \\ 2 School of Computer and Cognitive Sciences, Chukyo University \\ 101 Tokodachi, Kaizu-cho, Toyota, Aichi, 470-0393, Japan \\ \{hasegawa, jtoriwak\}@sccs.chukyo-u.ac.jp
}

\begin{abstract}
This paper presents a new method for extracting bronchus regions from $3 \mathrm{D}$ chest X-ray $\mathrm{CT}$ images based on structural features of the bronchus. This method enhances bronchial walls by applying a sharpening operation and segments each bronchial branch by recognizing the tree structure starting from the trachea. During the extraction process, the volumes of interest (VOI) which contains a bronchial branch currently being processed are defined. Region growing is performed only inside a VOI so that a bronchial branch is extracted by a suitable threshold value. The final bronchus region is obtained by unifying the extracted branches. The tree structure of the bronchus is also extracted simultaneously. The proposed method was applied to three cases of 3D chest X-ray CT images. The experimental results showed that the method significantly improved extraction accuracy. About $82 \%$ branches are extracted for 4 th-order bronchi, $49 \%$ for 5 th-order bronchi, and $20 \%$ for 6 th-order bronchi, compared to $45 \%, 16 \%$, and $3 \%$ by the previous method using the region growing method with a constant threshold value.
\end{abstract}

\section{Introduction}

The bronchus is a very important organ in diagnosis of the chest, since lung diseases such as lung cancers or emphysema are often concerned with the bronchus. Bronchial branches ramify to form a tree toward peripheral parts [1]. The bronchial lumen is filled with the air and surrounded by the bronchial wall whose CT value is relatively high. Therefore, the bronchus region can be roughly segmented by a region growing method based on a threshold value to CT values [2]. However, thinner bronchial branches after the 4-th branching level cannot be segmented correctly due to the partial volume effect (PVE) which increases CT values of thin bronchial lumen. The PVE causes mis-segmentation of branches during the region-growing process.

To improve segmentation accuracy, an optimal threshold value for each branch should be determined. Enhancement of bronchial walls is also required. 


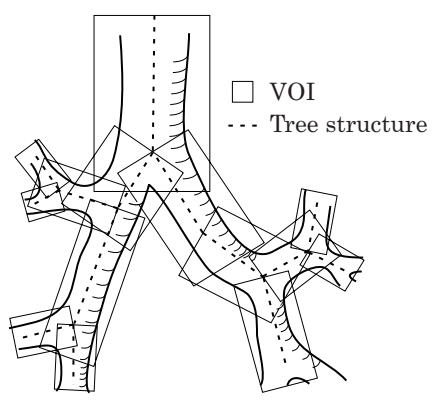

Fig. 1. Illustration of segmentation of the bronchus.

Researches have been reported on improvement of the extraction accuracy of the bronchus 3456. They use anatomical knowledge of the bronchus [3], adaptive thresholding by tracing cross sections of each bronchial branch [4], and the front propagation technique 6. However, they do not take enhancement of bronchial walls into account. In the proposed method, the concept of the volume of interest (VOI) is introduced to segment a bronchus region branch by branch and to enhance bronchial walls. The VOIs divide a given CT image into subimages (VOI images) that contain one bronchial branch in each of them (Fig. 1). For each VOI image, a sharpening operation based on the Laplacian of Gaussian (LoG) filter is applied so as to enhance a bronchial wall. An advantage of the proposed method is to provide the graph representation of the extracted bronchus tree simultaneously. The method is also applicable to other organs such as lung vessels.

In Section 2, we describe the extraction procedures of a bronchus region using VOIs. Section 3 shows extraction results for chest X-ray CT images.

\section{Method}

\subsection{Overview}

A bronchus region is extracted by tracing a bronchus tree using VOIs. Each bronchial region inside a VOI image is segmented sequentially from the trachea by extending a VOI in the running direction of the branch. When the bronchial branch bifurcates, child VOIs are placed on the child branches. A sharpening operation based on the LoG filter is applied to enhance the bronchial wall that is weakened by the PVE for each VOI image. During tracing a bronchial tree, simple anatomical knowledge of the bronchus described below is used;

Knowledge (i) each branch has two or three child branches,

Knowledge (ii) the diameters of branches become smaller with increasing bifurcation levels.

In the proposed method, prediction of branching directions of bronchi is not performed because there are many variations in branching pattern. 


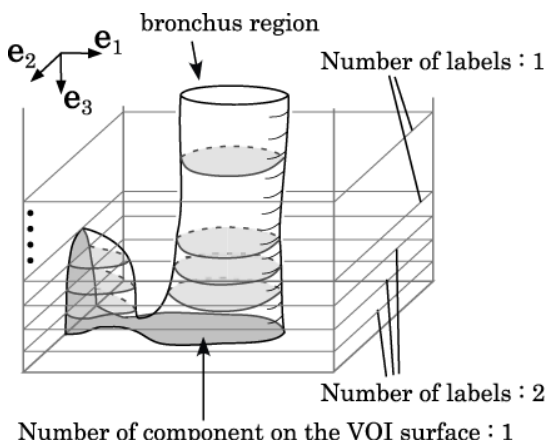

Number of component on the VOI surface : 1

Fig. 2. An example of the leak detection.

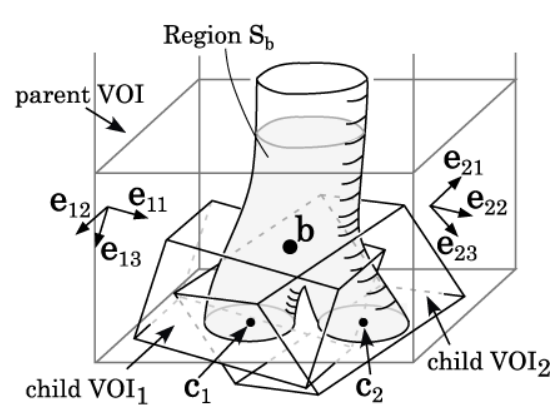

Fig. 3. Illustration of placing child VOIs.

A VOI determines a local processing area along with the direction of a bronchial branch. It is defined by three basis vectors, $\mathbf{e}_{1}, \mathbf{e}_{2}$, and $\mathbf{e}_{3}$ and the lengths along with the basis vectors, $l_{1}, l_{2}$ and $l_{3}$. The direction $\mathbf{e}_{3}$ is aligned to the running direction of the bronchial branch. We generate a VOI image where image resolution is isotropic by tri-cubic interpolation. The sharpening operation by the LoG filter is applied to enhance bronchial walls. The sharpened image $\mathbf{F}_{\text {sharpen }}$ of the VOI image $\mathbf{F}$ is given by

$$
\mathbf{F}_{\text {sharpen }}=\mathbf{F}-\beta \operatorname{LoG}(\mathbf{F}) \quad(\beta>0),
$$

where $\operatorname{LoG}(\mathbf{F})$ is an output of the LoG filter for an input $\mathbf{F}$, and $\beta$ is a coefficient of the sharpening operation.

\subsection{Processing Procedures}

The proposed method consists of the following seven steps.

\section{Step (1) Rough segmentation}

A bronchus region is roughly segmented by the region growing method developed by Mori et al. [2]. The threshold value $T_{i n i}$ determined by the Mori's method is used as the basic threshold value to segment each bronchial branch.

\section{Step (2) Placement of the VOI for the trachea}

The trachea region in the top (most head-slide) slice of a CT image is obtained from the bronchus region extracted in Step (1). The radius of the trachea $r_{t r a}$ is calculated from the number of pixels of the trachea region in the top slice. The center of gravity of the trachea $\mathbf{c}_{t r a}$ is also calculated. The VOI for the trachea is initially determined as a cube whose edge lengths are five times of $r_{t r a}$. The center point of the VOI is located at $\mathbf{c}_{t r a}$. The directions of the axes of the VOI are parallel to those of the CT image.

\section{Step (3) Segmentation of a bronchus region in the VOI}

For the current VOI, a bronchus region is extracted by applying the Mori's method. A suitable threshold value $T$ that satisfies the conditions (I) and (II) 
described in Step (4) is found by a binary search algorithm. The upper threshold limit $T_{\text {upper }}$ and the lower limit $T_{\text {lower }}$ are $T_{i n i}+100$ and -1200 H.U., respectively. In the growing process, two levees (planes) are set to prevent reversegrowing toward the parent and sibling branches. They are determined by the direction of the parent VOI $\mathbf{e}_{3}$ and the bifurcation point of the parent branch. No voxels beyond the levees are extracted.

\section{Step (4) Detection of bifurcation}

Bifurcation is detected by analyzing the number of connected components appearing on the surface of the VOI image. Extracted voxels on the surface are classified by three dimensional labeling process, and the number of voxels of each label $S_{i}\left(1 \leq i \leq N_{c}\right)$ is measured. The leakage beyond the bronchial wall is detected by checking $N_{c}$ and the number of components $N_{c}(j)\left(1 \leq j \leq l_{3}\right)$ on each cross section of the VOI image (Fig. 2). In the case of normal bifurcation, a bronchial branch never bifurcates backward to the direction $\mathbf{e}_{3}$, that is, $N_{c}(j) \leq N_{c}$. Hence, if $N_{c}$ is one or larger, i.e. possibility of bifurcation, the following conditions are checked;

Condition (I): $N_{c}<4$ (Knowledge (i)),

Condition (II): $N_{c}(j) \leq N_{c} \quad\left(1 \leq j \leq l_{3}\right)$.

If any of the conditions (I) and (II) is not satisfied, the leakage is detected (Fig. 2). When the leakage occur, $T_{\text {upper }}$ is decremented and Step (3) is processed again. If the above conditions are satisfied and $N_{c}$ takes two or three, a bifurcation (trifurcation) is detected and Step (7) is processed. If $N_{c}$ is zero, the extraction process is finished and bronchial branches after the current branch are not searched. Otherwise $\left(N_{c}=1\right)$, the direction of the VOI is checked (Step (6)) before extending the VOI (Step (5)).

\section{Step (5) Extension of the VOI}

The length of the VOI, $l_{3}$, is extended by one voxel and the VOI image is recalculated. After the extension, Step (3) is processed again.

\section{Step (6) Modification of the direction of the VOI}

When there is the difference between the direction of the VOI and that of the bronchial branch being currently processed, the direction of the VOI is modified so as to coincide with that of the branch.

\section{Step (7) Placement of child VOIs}

If a bifurcation is detected in Step (4), VOIs for the child branches of the currently extracted branch are placed by the following steps (Fig. 31). The mean radius of the extracted bronchus region $S_{b}$ in the current VOI $r=\sqrt{\#\left(S_{b}\right) \pi / l_{3}}$ is calculated, where $\#\left(S_{b}\right)$ is the number of the voxels of the region $S_{b}$. The branching point $\mathbf{b}$ is given as the center of gravity of the region $S_{b}$. The centers of gravity, $\mathbf{c}_{i}$, of components $S_{i}$ detected in Step (4) are also measured. The direction of a child VOI is set to $\mathbf{c}_{i}-\mathbf{b}$. The size of the child VOI $\left(l_{i 1}, l_{i 2}, l_{i 3}\right)$ are determined as $\left(5 r, 5 r,\left\|\mathbf{c}_{i}-\mathbf{b}\right\|\right)$. The bronchus region that have been extracted in the current VOI is added to the final bronchus region. Then, Steps (3)-(6) are repeated for extracting subsequent branches. 


\section{Experiment and Discussion}

We applied the proposed method to three chest X-ray CT images taken by multi detector-row CT scanners. The acquisition parameters of the images are; $512 \times$ 512 pixels in a slice image, 156 - 350 slices, 0.546 - $0.625 \mathrm{~mm}$ pixel spacing, $2.0 \mathrm{~mm}$ in X-ray beam width, and 1.0 - $2.0 \mathrm{~mm}$ slice spacing. Figure 4 shows examples of the 3D displays of the extracted regions by the proposed method and the Mori's method 2]. Figure 5 shows examples of the extracted tree structure for Case 1. An example of slice images processed by the sharpening operation is shown in Fig.6. It is found that the bronchial wall are effectively enhanced by the sharpening operation. Table 1 shows the number of extracted branches by the proposed method and the Mori's one. The method could extract 244 branches for Case 1, 123 for Case 2 and 83 for Case 3, while 101, 54 and 38 by the Mori's method, respectively. The extraction accuracies for the 3rd-, 4th-, and 5th-order bronchi were also evaluated (Table 2). About $82 \%$ branches were extracted for 4 th-order bronchi, $49 \%$ for 5 th-order bronchi, and $20 \%$ for 6 th-order bronchi, compared to $45 \%, 16 \%$, and $3 \%$ by the Mori's method. The computation time was about forty minutes per case (CPU: AMD Athlon 1900+).

As shown in Fig. 4 and Table [1] effectiveness of the sharpening operation and segmentation with an adaptive threshold value by using VOIs was revealed. Especially for Case 1, $98 \%$ of 4 th-order, $78 \%$ of 5 th-order, and $46 \%$ of 6 th-order bronchial branches are segmented. However, there are 15 leakages for Case 1, 6 for Case 2, and 5 for Case 3. The sharpening operation enhances not only bronchial walls but also voxels that are not bronchial walls, e.g., noise voxels. Since the coefficient $\beta$ for the sharpening operation closely relates to the occurrence of leakages, such leakages must be eliminated by post-processing. Branching pattern analysis may help to eliminate such leakages.

Table 1. Comparison of the number of bronchi and leakages (false branches) extracted by the proposed method and the Mori's method [2]. Terms "I" and "S" mean interpolation and sharpening, respectively.

\begin{tabular}{|c|c|c|c|c|c|c|c|c|}
\hline \multirow{2}{*}{ Case } & \multicolumn{5}{|c|}{ Number of extracted bronchi } & \multicolumn{3}{|c|}{ Number of leaks (false branches) } \\
\hline & $\begin{array}{l}\text { Propos } \\
\beta: 0.05\end{array}$ & $\begin{array}{c}\text { sed method } \\
0.15 \quad 0.3 \\
\end{array}$ & $\begin{array}{c}\text { Pres } \\
\text { w/o } \\
\text { I nor S } \\
\end{array}$ & $\begin{array}{l}\text { ous } \mathrm{m} \\
\mathrm{w} / \mathrm{I} \\
\end{array}$ & $\begin{array}{c}\text { ethod } \\
\text { w/ } \\
\text { I and S }\end{array}$ & $\begin{array}{l}\text { Proposed m } \\
\beta: 0.05 \quad 0.15\end{array}$ & $\begin{array}{c}\text { ethod } \\
0.3 \\
\end{array}$ & Previous method \\
\hline 1 & 222 & 244246 & 92 & 101 & 134 & $9(4) \quad 15(8)$ & $22(34)$ & $0(0)$ \\
\hline 2 & 101 & 123131 & 46 & 54 & 64 & $0(0) \quad 6(2)$ & 10(11) & $0(0)$ \\
\hline 3 & 65 & $83 \quad 90$ & 32 & 38 & 38 & $2(7) \quad 5(5)$ & $12(15)$ & $0(0)$ \\
\hline
\end{tabular}




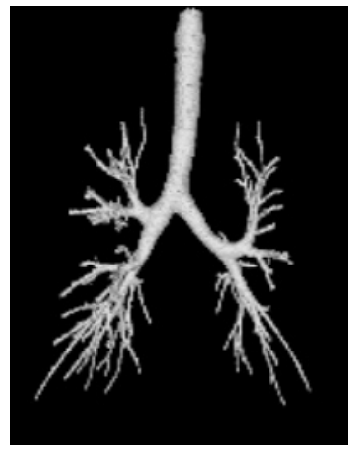

Case 1

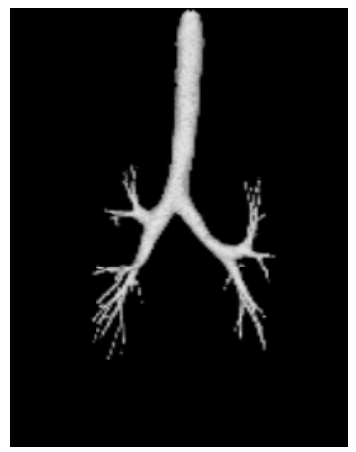

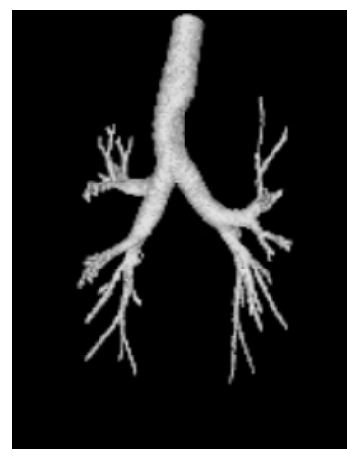

Case 2

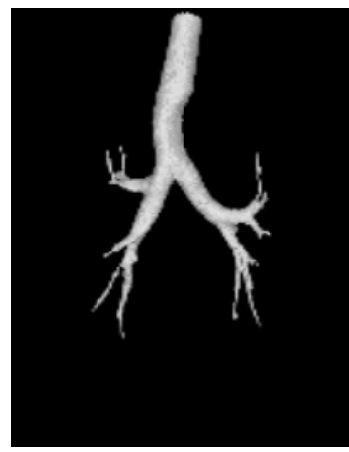

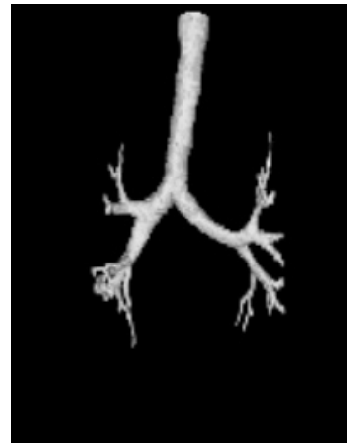

Case 3

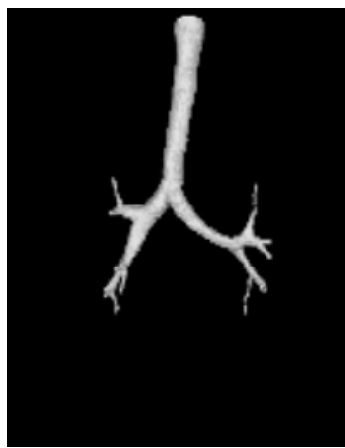

Fig. 4. Examples of the 3D displays of extraction results. (Upper row) proposed method, (Lower row) Mori's method [2].
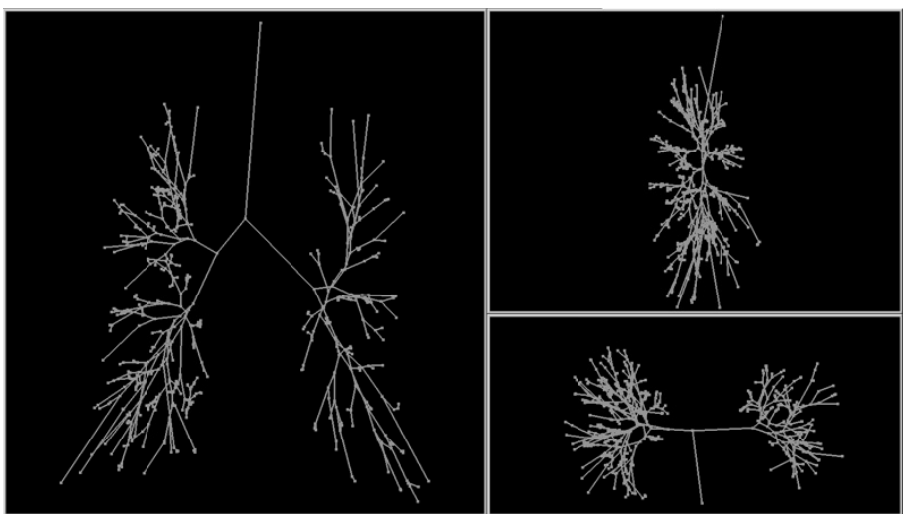

Fig. 5. Examples of the extracted tree structure of the bronchus for Case $1(\beta=0.15)$. The left-side, right-upper, and right-lower images are the images when seeing from the front, the right, and the top of the human body. 


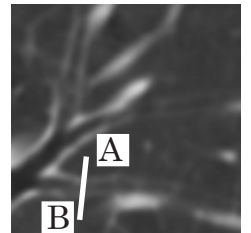

(a)

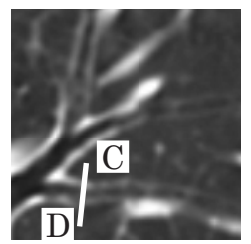

(c)

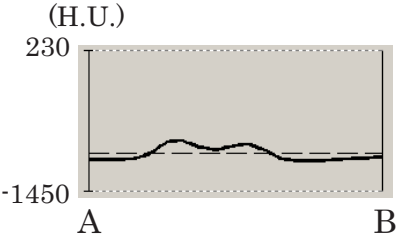

(b)

(H.U.)

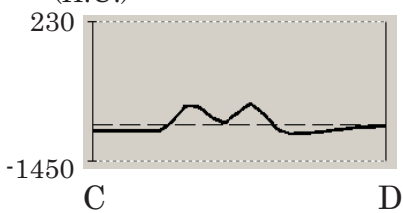

(d)

Fig. 6. An example of the sharpened image. (a) Original image, (b) Intensity profile from A to B in (a), (c) Sharpened image of (a), (d) Intensity profile from C to D.

Table 2. Comparison of the extraction accuracies for each order of branches by the proposed method and the Mori's method with interpolation and sharpening.

\begin{tabular}{|c|c|c|c|}
\hline \multirow{2}{*}{\multicolumn{2}{|c|}{ Case }} & \multicolumn{2}{|c|}{ Number of extracted bronchi } \\
\hline & & $\begin{array}{r}\text { Proposed method } \\
\beta: 0.15\end{array}$ & $\begin{array}{c}\text { Previous method } \\
\text { w/ I and S }\end{array}$ \\
\hline \multirow{3}{*}{1} & 4 th & $40 / 41(98 \%)$ & $30 / 41(73 \%)$ \\
\hline & 5 th & $64 / 82(78 \%)$ & $35 / 82(43 \%)$ \\
\hline & 6 th & $75 / 164(46 \%)$ & $12 / 164(7 \%)$ \\
\hline & 4 th & $34 / 42(81 \%)$ & $20 / 42(45 \%)$ \\
\hline & 5 th & $39 / 84(46 \%)$ & $4 / 84(5 \%)$ \\
\hline & 6 th & $18 / 168(11 \%)$ & $1 / 168(1 \%)$ \\
\hline \multirow{3}{*}{3} & 4 th & $28 / 42(67 \%)$ & $8 / 42(19 \%)$ \\
\hline & 5 th & $20 / 84(24 \%)$ & $0 / 84(0 \%)$ \\
\hline & 6 th & $2 / 168(2 \%)$ & $0 / 168(0 \%)$ \\
\hline
\end{tabular}




\section{Conclusion}

We developed a method for segmenting bronchial trees from 3D chest X-ray CT images by using structural features of the bronchus. This method extracted each bronchial branch by tracing the bronchial tree by using the VOIs. The bronchial walls were enhanced by the sharpening operation during segmentation. The proposed method significantly improved the accuracy of segmentation. However, there remains possibility of generating false branches due to leakages in the peripheral parts of the lung. Future work includes the further experiment using much larger number of CT images, development of the post-processing to eliminate leaked areas, and the reduction of computation time.

Acknowledgments. The authors thank Dr. S. Nawano of National Cancer Center Hospital East Japan, Dr. H. Natori of Sapporo Medical University, Dr. M. Mori of Sapporo Kousei Hospital, Dr. H. Takabatake of Minami-ichijyo Hospital, and Dr. H. Otsuji of Saisei Suita Hospital for providing the experiment samples. They also thank the members of our laboratory at Nagoya University for their collaboration. This work was supported in part by the Grant-In-Aid for Scientific Research for Private University High-Tech Research Center and 21st COE program provided by the Ministry of Education, Culture, Sports, Science and Technology of the Japanese government and the Grant-In-Aid for Cancer Research from the Ministry of Health, Welfare and Labor of the Japanese government.

\section{References}

1. F. H. Netter: Atlas of human anatomy -second edition, NOVARTIS, PLATE 190$193,1997$.

2. K. Mori et al.: Automated extraction and visualization of bronchus from 3D CT images of lung, Proc. of 1st International Conference on Computer Vision, Virtual Reality and Robotics in Medicine (CVRMed'95), pp. 542-548, 1995.

3. M. Sonka et al.: Rule-based detection of intrathoracic airway trees, IEEE Trans. on Medical Imaging, 15, 3, pp. 314-326, 1996.

4. F. Iseki et al.: Extraction of 3D tree structure of blood vessels in lung area from chest CT images, Proc. of CAR'98, pp. 45-50, 1998.

5. Y. L. Tsui et al.: Automated extraction of bronchus from 3D CT images of lung based on generic algorithm and 3D region growing, Proc. of SPIE on Medical Imaging 2000, 3979, pp. 906-916, 2000.

6. T. Schlathoelter et al.: Simultaneous segmentation and tree reconstruction of the airways for virtual bronchoscopy, Proc. of SPIE on Medical Imaging 2002, 4684, pp. 103-113, 2002. 